\title{
Cardiac Insufficiency: Understanding the Dimension of Advice
}

\author{
Andréa Brunelli Donnard* \\ Clinical Psychologist, Brazil
}

Submission: December 20, 2016; Published: January 05, 2017

*Corresponding author: Andrea Rodrigues Brunelli Donnard, Rua of Nightingales, 11 Hat Hill condo, Nova Lima, MG Brazil, Clinical psychologist and psycho oncologist; Tel: 55 (31)-99377-5805; Email: adonnard55@gmail.com

\begin{abstract}
Quality of life, in almost all the current literature reviewed; affirm that it is a set of conditions and situations that engulf the physical, spiritual, psychological, emotional well being, besides social relationships, family, friends, etc. If we analyze this affirmation, we can say that it is the daily life of every human being, healthy, productive, and active and that has internalized values like dignity and feelings of social belonging.

Congestive heart failure is a condition in which the heart is unable to pump blood into the bloodstream in sufficient quantity to meet the needs of the body. (I would like to remind you that I am not a cardiologist and the point of analysis and revision in this article of opinion is about the quality of life and the emotional processes and impacts on the human being). In developed countries 6 to $10 \%$ of the population over 65 is affected, and in the year following diagnosis the risk of death is around 35\%. It is here that we begin to interweave the healthy, autonomous individual, agent of his life, to the individual when faced with a limiting, chronic and serious diagnosis.
\end{abstract}

Keywords: Heart disease; Diagnoses; New lifestyle; Fears

\section{Introduction}

To be sick is to lose health integrity; to treat their illness (main objective of medicine) is to restore lost integrity, understood as biological, psychosocial and spiritual wellbeing. As defined by the United Nations (UN), as quality of life. Difficulty, healing, quality of life, integrity. Well, it seems like a cycle closes, if it really works this way forever. But we know that we are not creatures to program, in this way, we get sick, we fail as people (guilt for getting sick?), We fail as health professionals, and we will have chronic illnesses to understand and many times emergency admissions to hospitals and heart failure.

Lya Luft, in her book, The Fatal Side, 2012, will present us with the foreboding of an unexpected diagnosis: "do not tell me what happens, do not say that life goes on, that time helps, how much do I have Children and friends and a job to do. Not comfort me ... Do not say anything, I see well that the sun is still rising, (...) Do not comfort me: from my pain I know. "

In the world 23 million cases. In the United States: a major public health problem, 5.2 million cases, 300,000 annual deaths and 54,000 direct deaths."But the suffering is only intolerable when nobody cares." (Dame Cicely Sauders). And the initial time passed, the process of mourning, after all the life that was in the hand of the subject, at first is felt as taken, torn from its control, and that will require a time of elaboration, understanding that will reach him and everyone The members of the family and the community. So we review once again the concept, quality of life, illness, and one more important fact: adherence to the proposed treatment [1-3].

Most of the literature states that the goal of treatments is to prolong the patient's life and improve their quality of life. After leaving this sentence well highlighted because I will return to it, as my guiding line throughout this article, I will quote for the non-academic reader's knowledge what types of heart failure and its physical implications, adding emotional ones.

Acute Heart Failure: Occurs suddenly and is of great risk. It is usually the result of a myocardial infarction, or severe arrhythmia. It is a serious situation, and despite the rapid attendance, it is fatal.

Congestive heart failure is a physiological state in which the heart is unable to pump blood to the needs of the metabolizing 
tissues. It usually develops gradually and because it is a chronic situation, can lead to a long life, with limitations to the carriers.

Many are the causes, from congenital, to hyperthyroidism, Chagas' disease, aortic stenosis, ischemic heart disease, hypertensive cardiopathy, Some are symptoms of heart failure, noting that not all symptoms appear together at the same time, and it is important to note that they are not unique to this disease. They are: dyspnea (shortness of breath to physical exertion and lying down), cough, weakness, edema, abdominal pain, palpitation, dizziness and decreased enuresis. Tachycardia, jugular stasis and hepatomegaly citing here as an example for knowledge. When Francis Bacon states that "men seek in society, comfort, utility and protection", we can then analyze how before a diagnosis that will affect the heart of the individual, we will talk about prolongation and quality of life,

Heart: in the figurative sense, thirst for affectivity. Seat of all the feelings, experiences, palpitations of love, anxiety, broken hearts and much more that we have to list. The heart that shelters in the human being, carries a history, desires choices, professions, life. Fears, fear of death ... may be. The heart of the individual who up to that moment harbors in their emotions, the sense of belonging, utility, community. The certainty of possessing quality of life. Even if he did not know that he was lacking much more, but the autonomy and the command, assured him this certainty.

If there is time for diagnosis, he will not know, in the great part of the patients, to separate this heart of love and feelings from the hollow muscular organ of the vertebrates, which receives the blood from the veins and pushes it into the arteries. And the long road of chronic patients and their families, will be based in many cases on difficult perceptions of pain, suffering and the feeling of dependence, ignorance and insecurity. Health professionals are faced with issues that are not only conclusive, but questions of value and non-scientific. Such as: living longer or suffering less.... how to all quality of life if the individual is seen without autonomy, dignity, loss of productivity, social relationships, cultural and many times family.

Thus, in face of a patient with chronic heart failure, we should observe the group of physical symptoms, the treatment of the individual, but together with a multidisciplinary team, understand the dimension of suffering and care, which will be integral and continuous. Oferecidos to patients and relatives. Many will be the ways and means that can be used as help in adherence to treatment, and that he may live relieved of his physical, psychological and / or spiritual suffering.

\section{Conclusion}

We have done a brief report of an article that, based on a research with patients with heart failure, will be published later with all the data and graphs that accompany it. But what is important is that we can observe that the diagnosis of a chronic - evolutionary disease, such as heart failure, establishes an integral as sentence bond with the various professionals who assist it and its family. We analyze the behavior of the vision of the symptoms, but together we present the importance and value attributed to the heart and feelings signification, evolving to establish a link of difficulties to deal with symptoms, and adherence to the treatment.

The moment we are ready to understand, listen and recognize the patient, who suffers and speaks, we assure the word of its ethical dignity.

\section{References}

1. Bowby, John (2004) Loss - Sadness and Depression. São Paulo - Editora Martins Fontes. Brazil.

2. Lafayette, Maria Inalda (2007) Disease and Family - Family feeling in the process of illness, Recife, Fasa: Brazil.

3. Gicirelli Victor (2011) Religious and Non Religious Spirituality in Relation to Death Acceptance or Rejection. Death Stud 35(2): 124-146.

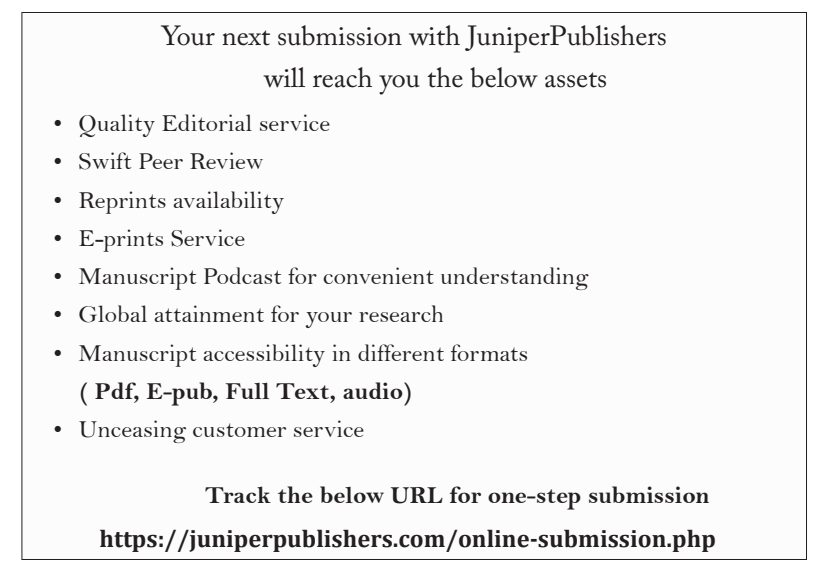

